\title{
Prevalence of incidentally discovered findings on brain MRI in adult Egyptian population
}

\author{
Dena Serag ${ }^{*}$ and Eman Ragab
}

\begin{abstract}
Background: Apparently, asymptomatic intracranial anomalies of potential clinical significance are becoming problematic, especially with the increase of use of brain MRI by clinicians and researchers. Performing MRI at higher resolution and stronger magnetic field and with more selective sequences has led to the detection of subtle brain anomalies that were not detected previously. Incidental findings are defined as previously undetected anomalies of potential clinical relevance that are unexpectedly detected and not related to the indication of the scan. Knowing about their existence would help clinicians to inform their patients of the potential risks and plan for long-term follow-up when indicated.

Results: Seven hundred fifty-three neurologically healthy subjects (389 males and 364 females) who underwent MRI scanning of the brain were screened for the presence of incidental findings, and the prevalence of each finding was recorded. Incidental findings were recorded in $11.7 \%$ of the subjects with meningiomas being the most commonly encountered finding (2.5\%) followed by vascular malformations (aneurysms, cavernomas) in about 2\% of the subjects. Meningiomas, cavernomas, and aneurysms had significantly higher prevalence in females than in males. The prevalence of neoplastic incidental findings increased with age, while the detection of non-neoplastic findings seemed to decrease with age.

Conclusion: Incidental findings were relatively common in the adult Egyptian population. The most common findings were meningiomas, followed by vascular malformations and arachnoids cysts. Knowing about their existence will help directing patients towards the proper specialist and plan for appropriate follow-up to avoid potential clinical hazards. It also raises the awareness of the importance of screening the brain scans while scanning the patient for other purposes like scanning the paranasal sinuses, orbits, and petrous temporal bones.
\end{abstract}

Keywords: Incidental brain findings, MRI

\section{Background}

Over the past decades, MRI brain imaging acquired a firm position in clinical practice and medical research as a powerful means of detecting both anatomic and functional information about the brain [1]. Subsequently, the detection of incidental findings (i.e., previously undetected anomalies of potential clinical relevance that are unexpectedly detected and not related to the indication of the

* Correspondence: denaserag@yahoo.com

Department of Radiodiagnosis, Faculty of Medicine, Menoufia University, Menoufia 32511, Egypt

\section{Springer Open}

scan) in large numbers of presumably neurologically healthy participants became a well-recognized and more frequent topic of concern $[2,3]$.

Knowing the chance of discovering incidental brain findings would help clinicians to inform their patients of the potential risks and plan for long-term follow-up when indicated $[4,5]$.

Previous studies investigated the prevalence incidental findings, such as brain tumors and vascular malformations, in healthy volunteers or in patients who underwent MRI scanning for various reasons [6, 7]. To date, few 
population-based studies have reported the occurrence of incidental brain findings [8].

Subclinical white matter ischemic changes such as white matter hyper-intensities and asymptomatic brain infarcts are not generally classified as incidental findings, the prevalence of which are known to be high in elderly age groups and increases with age [9-11]. They are usually considered to be a part of the normal aging process of the brain [11]; thus, they were not included in our study.

The current study reports on the prevalence of incidental findings of potential clinical significance on brain MRI in the adult Egyptian population (excluding white matter ischemic changes) detected by high-resolution brain MRI in 753 neurologically healthy subjects.

\section{Methods}

\section{Study population}

This retrospective observational study was conducted in the period from January 2017 to November 2019. It included 753 adult patients (389 \{51.7\%\} males and 364 $\{48.3 \%\}$ females), with a mean age of $\{ \pm S D\} 49.8$ years $\{ \pm 18.68\}$, age range (18-96 years). They were referred to radiology department for MRI scanning of the orbits, paranasal sinuses (PNS), or petrous temporal bones. Patients were referred with a variety of symptoms including nasal obstruction, sinus headache, proptosis, local orbital swellings, and hearing problems. Initial scans included axial FLAIR and axial T2WI of the whole brain. Upon spotting an incidental brain lesion during the initial review, the scan was extended following the standard brain MRI protocol described below. Inclusion criteria were the following: adult patients who were neurologically healthy with no clinical evidence of neurologic deficit. Exclusion criteria were the following: patients with incidental brain finding that might be related to patient's chief complaint and scans significantly compromised by motion artifacts. Out of the 830 scans initially reviewed, 753 patients were finally included in the study. The study was approved by the local Research Ethics Committee on 24 November 2019, reference number of approval: 191119-RAD-12. All patients included in this study gave written informed consent to participate in this research.

\section{Examination protocol}

MRI scans were performed using a 1.5-Tesla unit (TOSHIBA, Excelart Vantage). Patients were scanned in the supine position using a 16-channel head coil. Before MR examination, patients were asked to remove any metallic objects around the head. Five hundred thirty-five patients received intravenous contrast agents according to the main indication of the scan.
The basic MRI scan protocol of the brain included T1WI $(\mathrm{TR}=464 \mathrm{~m} / \mathrm{s}, \mathrm{TE}=12 \mathrm{~m} / \mathrm{s}, \mathrm{FOV}=24 \mathrm{~cm}, \mathrm{NS}=$ 20 , matrix $=192 \times 352$ pixels $)$, T2WI $(T R=4300 \mathrm{~m} / \mathrm{s}$, $\mathrm{TE}=105 \mathrm{~m} / \mathrm{s}$, matrix $=256 \times 448$ pixels), and FLAIR $(\mathrm{TR}=7200 \mathrm{~m} / \mathrm{s}, \quad \mathrm{TE}=100 \mathrm{~m} / \mathrm{s}, \quad \mathrm{TI}=2000 \mathrm{~m} / \mathrm{s}$, matrix $=$ $192 \times 352$ pixels) were obtained in the axial plane using 5 - $\mathrm{mm}$ contiguous scans. Sagittal T1WI $(\mathrm{TR}=540 \mathrm{~m} / \mathrm{s}$, $\mathrm{TE}=17 \mathrm{~m} / \mathrm{s}, \mathrm{FOV}=25.6 \mathrm{~cm}$, matrix $=192 \times 320$ pixels, $\mathrm{ST}=4.6 \mathrm{~mm})$, coronal $\mathrm{T} 2 \mathrm{WI}(\mathrm{TR}=4280 \mathrm{~m} / \mathrm{s}, \mathrm{TE}=105$ $\mathrm{m} / \mathrm{s}, \quad F O V=24 \mathrm{~cm}, \quad$ matrix $=192 \times 448$ pixels, $\quad \mathrm{ST}=6$ $\mathrm{mm})$, and DWI $(b=1000, \mathrm{TR}=6800 \mathrm{~m} / \mathrm{s}, \mathrm{TE}=120 \mathrm{~m} / \mathrm{s}$, $\mathrm{FOV}=26 \times 26 \mathrm{~cm}, \quad$ matrix $=128$ pixels, $\mathrm{ST}=5.3 \mathrm{~mm}$ ) were also obtained.

\section{Assessment of incidental findings}

Images were transferred by computer network to workstation (Aze Virtual Place FujinRaijin 310) on which images were carefully examined. A trained radiologist (with more than 10 years of experience in neuroradiology) examined the scans and recorded the presence of incidental findings. Patients having multiple similar findings were counted as one finding (e.g., multiple meningiomas), while patients having two different findings were counted separately.

Seven patients required referral to specialists (neurologist, oncologist) (5 patients with subdural collections " 2 of which underwent surgical evacuation," 1 patient with suspicious low-grade glioma (DENT), and 1 patient with suspicious metastatic deposits). Diagnosis of neoplastic lesions was based on the imaging findings and no histopathologic confirmation was obtained.

Nineteen patients with meningiomas, 6 patients with aneurysms, 9 patients with pituitary macroadenomas, and 11 patients with arachnoid cysts were also referred to specialists (neurologist, internist) for further assessment and long-term follow-up.

The rest of the patients were informed about their unexpected abnormalities and reassured.

Anatomic variants of no clinical significance like cavum septum pellucidum and lateral ventricles asymmetry, as well as empty sella turcica and dilated Virchow-Robin spaces (VRS) were not included in the study. The current study did not include white matter hyper-intensities or lacunar infarcts either, being prevalent in the old age group and considered by most authors to be a part of the normal aging process of the brain.

\section{Statistical analysis}

Results were analyzed using an IBM compatible personal computer with SPSS statistical package version 23 (SPSS Inc. Released 2015. IBM SPSS Statistics for Windows, version 23.0, Armnok, NY: IBM Corp).

Data was expressed in number (No), percentage (\%), mean $(\mathrm{x})$, and standard deviation (SD). $Z$ test was used 
to compare two proportions in two groups. Two-sided $P$ value of $<0.05$ was considered statistically significant.

\section{Results}

This study included 753 adult patients, 389 males (51.7\%) and 364 females (48.3\%). Their ages ranged from 18 to 96 years (mean $\{ \pm S D\}=49.8 \pm 18.68$ years). Incidental brain findings of potential clinical relevance were reported in 88 patients $(11.7 \%)$ with 96 recorded anomalies. Eighty patients had a single anomaly and 8 patients had 2 anomalies. Forty-nine anomalies (51\%) were found in women while 47 anomalies (49\%) were found in men. Table 1 showed the prevalence of each recorded incidental finding. Figures 1 and 2 showed a selection of the incidental findings that were encountered in the current study. Among the recorded findings, meningiomas were the most common (19 meningiomas, 2.5\%) with a mean longest axis of $15 \mathrm{~mm}$. All of them were supratentorial in location (8 convexity meningiomas, 5 falcine meningiomas, 4 sphenoid wing meningiomas, and 2 sub-frontal meningiomas).

Table 1 The prevalence of each recorded incidental finding in 753 MRI scans

\begin{tabular}{ll}
\hline Abnormality & No. $(\%)$ \\
\hline Vascular malformations & \\
- Aneurysm & $6(0.8 \%)$ \\
- Cavernoma & $9(1.19 \%)$ \\
- Developmental venous anomaly & $3(0.4 \%)$ \\
- Vertebro-basilar dolicoectasia & $3(0.4 \%)$ \\
Benign tumors & \\
- Meningioma & $19(2.5 \%)$ \\
- Shwannoma & $2(0.26 \%)$ \\
- Intracranial lipoma & $7(0.92 \%)$ \\
- Pituitary macro-adenoma & $9(1.2 \%)$ \\
Malignant tumors & \\
- Metastasis & $1(0.13 \%)$ \\
- DENT & $1(0.13 \%)$ \\
Intracranial cysts & \\
- Arachnoid cyst & $11(1.46 \%)$ \\
- Epidermoid cyst & $3(0.4 \%)$ \\
- Dermoid cyst & $1(0.13 \%)$ \\
- Choroidal fissure cyst & $2(0.26 \%)$ \\
- Choroid plexus cyst & $2(0.26 \%)$ \\
Other findings & \\
- Chiari I malformation & $6(0.8 \%)$ \\
- Corpus callosum agenesis & $3(0.4 \%)$ \\
- Dandy walker variant & $2(0.26 \%)$ \\
\hline
\end{tabular}

Vascular malformations were relatively common in the current study found in 21 participants (2.7\%). Among the encountered vascular malformations, cavernomas were the most prevalent, found in 9 participants (1.2\%) (most of them were found in right parietal lobe "6 cases, $0.8 \%$ "), followed by aneurysms which were detected in 6 cases $(0.8 \%)$. All aneurysms were found in the anterior circulation (A-com) with a mean diameter of $6 \mathrm{~mm}$. None of them exceeded $10 \mathrm{~mm}$ in diameter.

Intracranial cysts were also relatively common in our study, found in 22 cases (2.9\%). Arachnoid cysts were the most prevalent, detected in 11 participants $(1.46 \%)$, with a mean longest diameter of $21 \mathrm{~mm}$. Most of them (7 cases, $0.9 \%)$ were supratentorial. Cerebellopontine angle epidermoid cysts were detected in 3 cases $(0.4 \%)$.

Pituitary macroadenomas were detected in 9 cases (1.2\%). Vestibular schwannomas had a prevalence of $0.26 \%(2$ cases).

In the current study, one possible malignant low-grade brain tumor (DENT) was detected that was not histologically confirmed. In another patient, an enhancing heterogeneous mass within the 4th ventricle was detected, and by revising patient's history, it was found that he had been treated for bronchogenic carcinoma (metastatic deposit).

Congenital malformations were detected in 11 cases (1.46\%). Among the participants, Chiari I malformation was the most prevalent congenital malformation, detected in 6 participants $(0.8 \%)$. Subdural collections were detected in 5 cases $(0.66 \%)$ (bilateral in 2 cases and unilateral in 3 cases).

The prevalence of meningiomas was significantly higher in females than in males (female to male ratio $=2.1: 1$ ) $(P<0.001)$. Aneurysms and cavernomas were also more prevalent in females (male to female ratio $=2: 1$ for both lesions). While for pituitary macroadenomas and arachnoid cysts, there was no significant gender predilection. Table 2 and Fig. 3 summarized the male to female ratio of the most commonly detected abnormalities.

Seventy eight percent (78\%) of non-neoplastic lesions were found in the age group 18-44 years. This percentage was significantly higher than the neoplastic lesions recorded in the same age group $(12.2 \%)\left(P^{<} 0.001\right)$. On the other hand, $58.5 \%$ of the neoplastic lesions were found in patients aged 45-59 years which was significantly higher than of the non-neoplastic lesions detected in the same age group $(7.3 \%)\left(P^{<} 0.001\right)$.

Table 3 and Fig. 4 showed the prevalence of neoplastic and non-neoplastic incidental findings in different age groups.

\section{Discussion}

Over the past decades, brain MRI has gained more significance in clinical practice and medical research. Its 


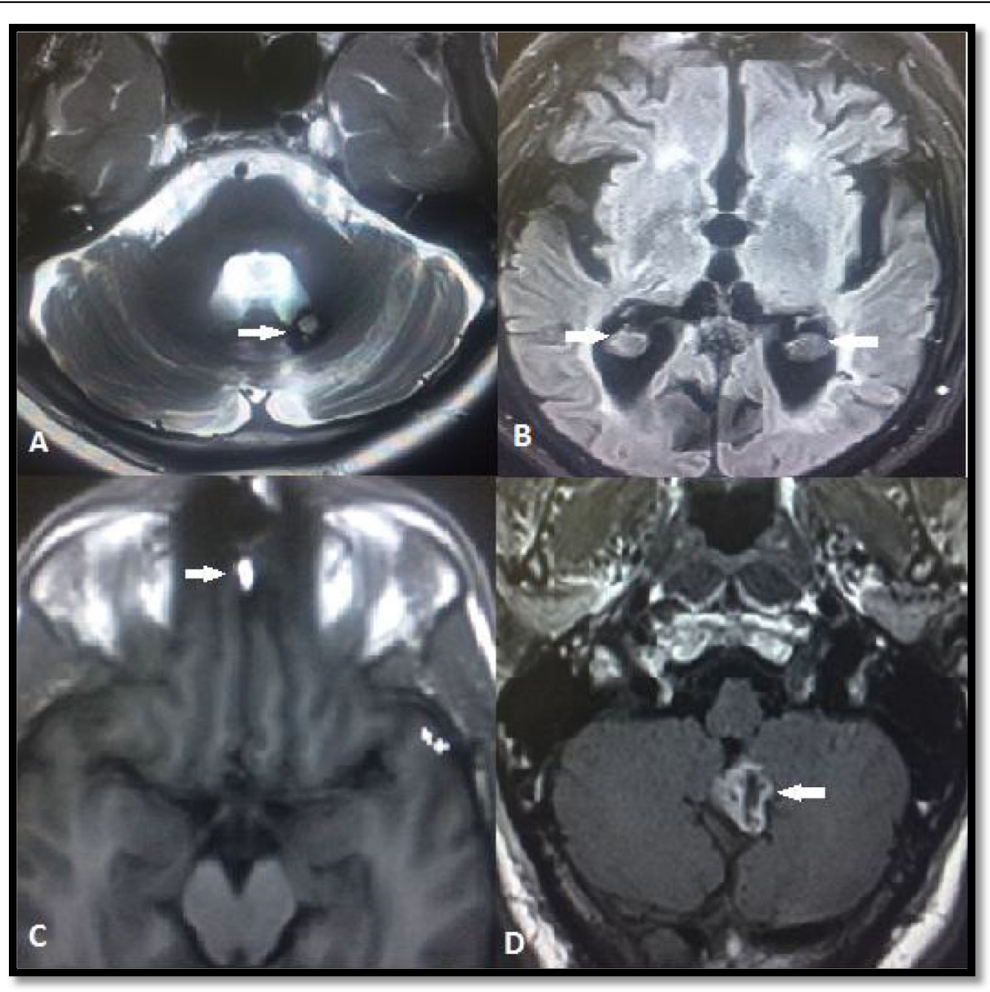

Fig.1 A selection of the incidental findings detected in our study. a Axial T2WI of the posterior fossa showing a left cerebellar hemisphere cavernoma (arrow). b Axial FLAIR image showing bilateral choroid plexus cysts (arrows). c Axial T1WI showing small inter-hemispheric lipoma (arrow). d Post contrast axial T1WI of the posterior fossa showing a heterogeneously enhancing mass in the 4th ventricle (metastasis) (arrow)

rapidly growing technical advances and increased availability led to widespread use. Subsequently, reporting the presence of incidental findings has become a well-recognized and more frequent topic of concern [12, 13]. Many populationbased studies have been carried out to investigate the prevalence rate of incidental brain findings of potential clinical relevance in general population, like the Rotterdam study carried out in Netherlands in 2005 [3]. To our knowledge, no previous data has been published about the prevalence of incidental brain findings in Egyptian population.

In the current study, a retrospective analysis of MRI scans for 753 neurologically healthy subjects (389 males "51.7\%" and 364 females "48.3\%") was performed. Their ages ranged from 18 to 96 years. Incidental brain findings of potential clinical relevance were reported in 88 (11.7\%) patients. These results were consistent with the results recorded by Bos et al. [12] in a population-based study. They scanned 5800 participants and found that incidental findings were detected in about $10 \%$ of the participants.

The results in the current study were higher than the results obtained by Vernooij et al. [3]. They held a population based study in Rotterdam, Netherlands, in 2005 on 2000 participants and reported a prevalence rate of $5.8 \%$ for incidental findings (excluding the vascular white matter lesions).
Among the recorded findings, meningiomas were the most common $(2.5 \%)$ with a mean longest axis of 15 $\mathrm{mm}$. These results were consistent with the results recorded by Bos et al. [12]. They recorded a $2.4 \%$ prevalence rate for meningiomas with a mean size of the longest axis $=13 \mathrm{~mm}$. Vernooij et al. [3] recorded a lower prevalence rate of for meningiomas $(0.9 \%)$.

Vascular malformations were recorded in $2.7 \%$ of the patients. Sandeman et al. [14] reported close findings, about $2 \%$.

Aneurysms were detected in $0.8 \%$ of the patients. These findings were close to the results obtained by Tsushima et al. [15]. They scanned 1113 adult participants aged $18-84$ years and reported a $0.62 \%$ prevalence rate for aneurysms. Vernooij et al. [3] reported a higher prevalence rate for aneurysms, about $1.8 \%$.

Arachnoids cysts were also relatively common in the current study detected in $1.4 \%$ of the patients. Vernooij et al. [3] reported a similar prevalence rate, about $1.1 \%$.

In the current study, 2 possible malignant tumors were found (one metastatic deposit and one low-grade glioma DENT), representing $0.26 \%$ of the patients. Onizuka et al. [16] reported a $0.3 \%$ prevalence rate for malignant brain tumors. 


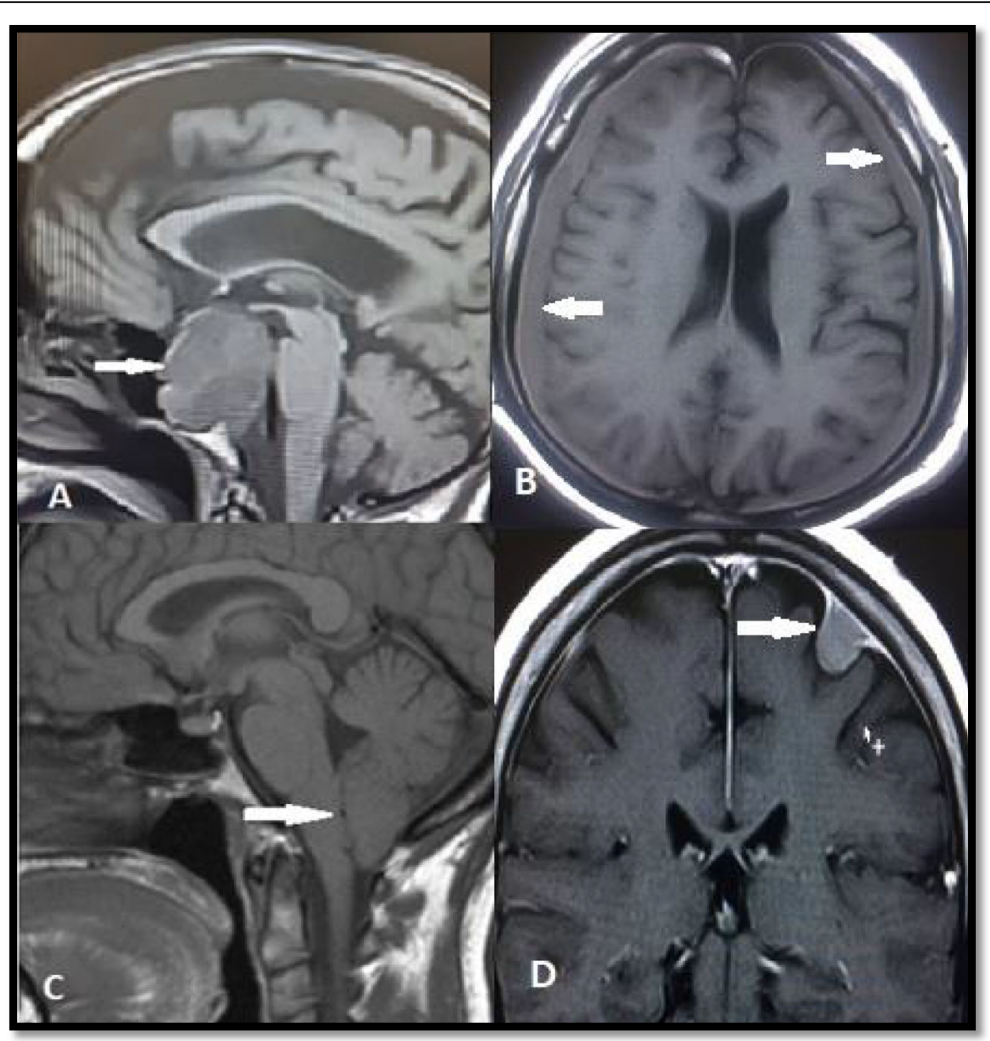

Fig. 2 A selection of the incidental findings detected in our study. a Sagittal T1WI showing a large pituitary macroadenoma with suprasellar extension (arrow). b Axial T1WI showing bilateral subdural hygromas (arrows). c Sagittal T1WI showing downward herniation of the cerebellar tonsils, more than $5 \mathrm{~mm}$ below the level of foramen magnum (Chiari I malformation) (arrow). $\mathbf{d}$ Post contrast coronal T1WI showing convexity meningioma) (arrow)

Fifty one percent (51\%) of the incidental findings in the current study were detected in women, while $49 \%$ were detected in men. Bos et al. [12] reported a slightly higher female predilection (55.7\% in women and $44.3 \%$ in men). The prevalence of meningiomas was significantly higher in females than in males (female to male ratio $=2.1: 1$ ). Bos et al. [12] reported similar results for meningiomas with a female to male ratio of 2.3:1.

Table $\mathbf{2}$ The male to female ratio of the most commonly detected abnormalities

\begin{tabular}{|c|c|c|c|c|}
\hline \multirow[t]{2}{*}{ Abnormality } & \multicolumn{2}{|c|}{ Male $(N=43)$} & \multicolumn{2}{|c|}{ Female $(N=45)$} \\
\hline & No. & $\begin{array}{l}\% \text { of affected } \\
\text { males }\end{array}$ & No. & $\begin{array}{l}\% \text { of affected } \\
\text { females }\end{array}$ \\
\hline -Aneurysm & 2 & 5.88 & 4 & 8.88 \\
\hline -Arachinoid cyst & 4 & 11.76 & 7 & 15.55 \\
\hline -Meningioma & 6 & 17.64 & 13 & 28.88 \\
\hline -Cavernoma & 3 & 8.82 & 6 & 13.33 \\
\hline $\begin{array}{l}\text {-Pituitary } \\
\text { macroadenoma }\end{array}$ & 4 & 11.76 & 5 & 11.11 \\
\hline
\end{tabular}

Aneurysms were also more prevalent in females (female to male ratio $=2: 1$ ). Bos et al. [12] got similar results.

Seventy-eight percent (78\%) of non-neoplastic lesions were found in the age group 18-44 years. This percentage was significantly higher than the neoplastic lesions recorded in the same age group. On the other

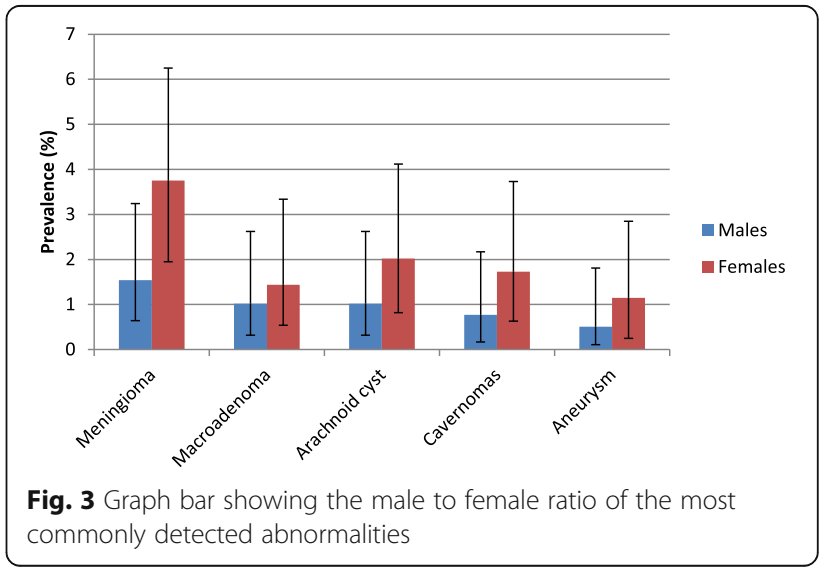


Table 3 The prevalence of neoplastic and non-neoplastic incidental findings in different age groups

\begin{tabular}{lllll}
\hline Age group & $\begin{array}{l}\text { Neoplastic } \\
(n=39) \\
\text { No. }(\%)\end{array}$ & $\begin{array}{l}\text { Non neoplastic } \\
(n=57) \\
\text { No. }(\%)\end{array}$ & $P$ value & $\begin{array}{l}P \text { value } \\
\text { of Z test }\end{array}$ \\
\hline $18-44$ y & $5(12.2)$ & $43(78.2)$ & $<0.001$ & $<0.001$ \\
$45-59$ y & $24(58.5)$ & $4(7.3)$ & & $<0.001$ \\
$60-74$ y & $9(22.0)$ & $5(9.1)$ & 0.139 \\
$75-99$ y & $3(7.3)$ & $3(5.5)$ & & 0.947 \\
\hline
\end{tabular}

hand, $58.5 \%$ of the neoplastic lesions were found in patients aged 45-59 years which was significantly higher than of the non-neoplastic lesions detected in the same age group. Morris et al. [5] carried out a systematic review and meta-analysis on 10,799 subjects from 6 different studies. They found that the prevalence of neoplastic incidental findings increased with age while the prevalence of non-neoplastic findings seemed to decrease with age.

\section{Limitations}

Some patients were excluded due to lack of detailed clinical history. We were not able to make sure that the patient's complaint is not related to the detected incidental finding.

In patients diagnosed with potentially malignant lesions (2 patients), final histopathologic diagnosis was not available.

\section{Conclusion}

Incidental findings were relatively common in the adult Egyptian population. The most common findings were

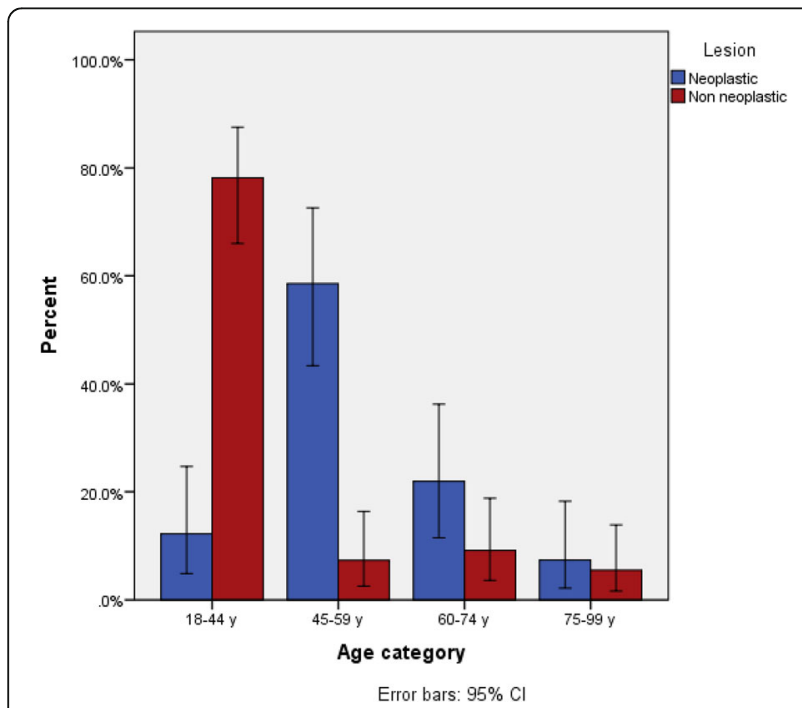

Fig. 4 Graph bar showing the prevalence of neoplastic and nonneoplastic incidental findings in different age groups (with 95\% confidence interval) meningiomas, followed by vascular malformations and arachnoids cysts. Knowing about their existence will help directing patients towards the proper specialist and plan for appropriate follow-up to avoid potential clinical hazards. It also raises the awareness of the importance of screening the brain scans while scanning the patient for other purposes like scanning the paranasal sinuses, orbits, and petrous temporal bones.

\section{Abbreviations}

MRI: Magnetic resonance imaging; DENT: Dysembryoplastic neuroepithelial tumor; VRS: Virchow-Robin spaces; FOV: Field of view; TI: Time of inversion;

FLAIR: Fluid attenuation inversion recovery

\section{Acknowledgments \\ There is no acknowledgment.}

\section{Authors' contributions}

DS and ER contributed equally to study design, data collection, analysis, and interpretation of results. All authors read and approved the final manuscript.

\section{Funding}

There is no funding.

Availability of data and materials

Data will be available upon request via contacting the corresponding author.

\section{Ethics approval and consent to participate}

This study was approved by the Research Ethics Committee of the Faculty of Medicine at Menoufia University in Egypt on 24 November 2019, reference number of approval: 191119-RAD-12. All patients included in this study gave written informed consent to participate in this research.

\section{Consent for publication}

All patients included in this research gave written informed consent to publish the data contained within this study.

\section{Competing interests}

The authors declare that they have no competing interests.

Received: 1 February 2020 Accepted: 15 April 2020

Published online: 23 April 2020

\section{References}

1. Illes J, Kirschen M, Edwards E, Bandettini P, Cho M, Ford P, Glover G, Kulynych J, Macklin R, Michael B, Wolf S, Grabowski T, Seto B (2008) Practical approaches to incidental findings in brain imaging research. Neurology 70 : 384-390

2. Salman R, Whiteley W, Warlow C (2007) Screening using whole-body magnetic resonance imaging scanning: who wants an incidentaloma? J Med Screen 14:2-4

3. Vernooij M, Ikram M, Tanghe H, Vincent A, Hofman A, Krestin G, Niessen W, Breteler M, Lugt A (2007) Incidental findings on brain MRI in the general population. N Engl J Med 357:1821-1828

4. Illes J, Kirschen M, Edwards E, Stanford L, Bandettini P, Cho M, Ford P, Glover G, Kulynych J, Macklin R, Michael D, Wolf S (2006) Ethics: incidental findings in brain imaging research. Science 311:783-784

5. Morris Z, Whitely W, Longstreth W, Weber F, Lee Y, Tsushima Y, Alphs H, Ladd S, Warlow C, Wardlaw J, Salman R (2009) Incidental findings on brain magnetic resonance imaging: systematic review and meta-analysis. BMJ 10: $1136-1143$

6. Glasmacher S, Thomas H, Stirland L, Wilkinson T, Lumsden J, Langlands G, Waddell B, Holloway G, Thompson G, Pal S (2019) Incidental findings identified on head MRI for investigation of cognitive impairment: a retrospective review. Dement Geriatr Cogn Disord 48(3):123-130

7. Weber $\mathrm{F}$, Knopf $\mathrm{H}$ (2006) Incidental findings in magnetic resonance imaging of the brains of healthy young men. J Neurol Sci 240:81-84

8. Shuyuan L, Fang F, Cui M, Jiang Y, Wang YX, Tian W, Fan M, Yuan Z, Chen J, Yang Q, Xue F, Wang J, Lu M, Wang X, Chen X, Jin L, Ye W (2019) Incidental 
findings on brain MRI among Chinese at the age of 55-65 years: the Taizhou Imaging Study. Sci Rep 9:464-471

9. Vermeer S, Koudstaal P, Oudkerk M, Hofman A, Breteler M (2002) Prevalence and risk factors of silent brain infarcts in the population-based Rotterdam Scan Study. Stroke 33:21-25

10. De Leeuw F, De Groot J, Achten E, Oudkerk M, Ramos L, Heijboer R, Hofman A, Jolles J, van Gijn J, Breteler M (2001) Prevalence of cerebral white matter lesions in elderly people: a population based magnetic resonance imaging study. J Neurol Neurosurg Psychiatry 70:9-14

11. De Groot J, De Leeuw F, Oudkerk M, Hofman A, Jolles J, Breteler M (2000) Cerebral white matter lesions and depressive symptoms in elderly adults. Arch Gen Psychiatry 57:1071-1076

12. Bos D, Poels M, Adams H, Akoudad S, Cremers L, Zonneveld H, Hoogendam Y, Verhaaren B, Verlinden V, Verbruggen J, Peymani A, Hofman A, Krestin G, Vincent A, Feelders R, Koudstaal P, Van der Lugt A, Ikram M, Vernooij M (2016) Prevalence, clinical management, and natural course of incidental findings on brain MR images: the population-based Rotterdam Scan Study. Radiology 281:507-515

13. Shoemaker J, Holdsworth M, Aine C, Calhoun V, De La Garza R, Feldstein Ewing S, Hayek R, Mayer A, Kiehl K, Petree L, Sanjuan P, Scott A, Stephen J, Phillips J (2011) A practical approach to incidental findings in neuroimaging research. Neurology 77(24):2123-2127

14. Sandeman E, Hernandez C, Morris Z, Bastin M, Murray C, Gow A, Corley J, Henderson R, Deary I, Starr J, Wardlaw J (2013) Incidental findings on brain MR imaging in older community-dwelling subjects are common but serious medical consequences are rare: a cohort study. PLoS One 15(8):1467-1471

15. Tsushima Y, Taketomi A, Endo K (2005) Prevalence of abnormal findings on brain magnetic resonance (MR) examinations in adult participants of brain docking. BMC Neurol 5:18-24

16. Onizuka M, Suyama K, Shibayama A, Hiura T, Horie N, Miyazaki H (2001) Asymptomatic brain tumor detected at brain check-up. Neurologia MedicoChirurgica (Tokyo) 41:431-434

\section{Publisher's Note}

Springer Nature remains neutral with regard to jurisdictional claims in published maps and institutional affiliations.

\section{Submit your manuscript to a SpringerOpen ${ }^{\circ}$ journal and benefit from:}

- Convenient online submission

- Rigorous peer review

- Open access: articles freely available online

High visibility within the field

- Retaining the copyright to your article

Submit your next manuscript at $\boldsymbol{\nabla}$ springeropen.com 